\title{
Real-world Case Series of iStent or iStent inject Trabecular Micro-Bypass Stents Combined with Cataract Surgery
}

David Manning

Received: July 19, 2019 / Published online: August 17, 2019

(C) The Author(s) 2019

\section{ABSTRACT}

Introduction: This real-world retrospective case series assessed 12-month effectiveness and safety following implantation of iStent ${ }^{\circledR}$ or iStent inject $^{\circledR}$ trabecular micro-bypass with cataract surgery.

Methods: Consecutive patients were implanted with either iStent (comprising 1 stent) or iStent inject (comprising 2 stents), together with cataract surgery. Most patients had primary openangle glaucoma. Effectiveness outcomes through 12 months included intraocular pressure (IOP) and glaucoma medications. Proportional analyses were completed for eyes achieving IOP $\leq 18 \mathrm{mmHg}$, IOP $\leq 15 \mathrm{mmHg}$, or IOP reduction $\geq 20 \%$ from preoperative; and eyes on 0 medications or $\geq 2$ medications. Safety outcomes included adverse events, secondary surgeries, visual acuity, and visual fields. Results: This analysis included 137 eyes (67 iStent, 70 iStent inject) with cataract and mild to moderate glaucoma or ocular hypertension. Over 73\% of eyes in both groups had early disease, and $\sim 22 \%$ had prior glaucoma surgery.

Enhanced digital features To view enhanced digital features for this article go to https://doi.org/10.6084/ m9.figshare.9302336.

D. Manning ( $\varangle)$

Hunter Cataract and Eye Centre, Charlestown, NSW, Australia

e-mail: ehdkmanning@hotmail.com
At 12 months postoperatively, mean IOP decreased from $18.4 \pm 4.2 \mathrm{mmHg}$ to $14.2 \pm$ $2.5 \mathrm{mmHg}$ in iStent eyes $(p<0.0001)$, and from $20.4 \pm 5.6 \mathrm{mmHg}$ to $14.4 \pm 2.1 \mathrm{mmHg}$ in iStent inject eyes $(p<0.0001)$. The IOP reduction was significantly greater for iStent inject eyes than iStent eyes $(6.0 \mathrm{mmHg}$ versus $4.2 \mathrm{mmHg}$ reduction, $p=0.034)$. Both groups had high proportions of patients achieving the 12-month IOP endpoints, although consistently greater proportions reached these endpoints after iStent inject than iStent: $95.7 \%$ versus $92.5 \%$ had IOP $\leq 18 \mathrm{mmHg}$, respectively; $74.3 \%$ versus $71.6 \%$ had IOP $\leq 15 \mathrm{mmHg}$, respectively; and $68.6 \%$ versus $62.7 \%$ had IOP reduction $\geq 20 \%$ from baseline, respectively. Mean medication burden at 12 months decreased from $1.8 \pm 0.7$ to $0.3 \pm 0.5$ medications in iStent eyes $(84.0 \%$ reduction, $p<0.0001)$, and from $1.3 \pm 0.9$ to $0.1 \pm 0.3$ medications in iStent inject eyes $(94.7 \%$ reduction, $p<0.0001)$. Significantly more iStent inject eyes were medication-free at 12 months than iStent eyes $(92.9 \%$ versus $76.1 \%$ medication-free, respectively; $p=0.0068$ ). Favorable safety included few adverse events, no secondary glaucoma surgeries, and stable visual acuity and visual fields in both groups.

Conclusion: Significant and safe IOP and medication reductions were achieved through 12 months after iStent or iStent inject implantation with cataract surgery. iStent inject eyes 
had greater absolute IOP reduction and more eyes medication-free versus iStent eyes.

Funding: Glaukos Corporation.

Keywords: Cataract; Glaucoma; Intraocular pressure; iStent; iStent inject; Microinvasive glaucoma surgery (MIGS); Second-generation; Stent; Trabecular micro-bypass

\section{INTRODUCTION}

Glaucoma is the leading cause of irreversible blindness, affecting nearly 80 million people worldwide [1]. If untreated, glaucoma results in vision loss that is progressive, permanent, and inevitable. Current glaucoma medications, laser treatments, and surgeries aim to reduce intraocular pressure (IOP), the only treatment strategy proven to limit glaucoma development and progression. Medications are effective and reasonably safe, and they usually are the first step in treating glaucoma patients. However, medications can be associated with substantial side effects [2-4], ocular surface damage [5-7], financial costs [8-14], and limited effectiveness due to nonadherence in an estimated $30-80 \%$ of patients $[15,16]$. Laser trabeculoplasty is modestly effective in reducing IOP, but its effectiveness wanes over time [17]. Standard glaucoma surgeries such as trabeculectomy and tube shunt implantation are highly effective in reducing IOP, but they have formidable safety risks, including significant vision loss and the need for reoperation, that preclude their widespread use, especially in milder glaucoma cases that may not warrant the risks of such surgeries [18-20]. Between these two ends of the treatment spectrum, there formerly was a large unaddressed middle ground, in which effective and safe intervention was not available.

Over the past 10 years, micro-invasive glaucoma surgery (MIGS) procedures have greatly augmented surgeons' available interventions for these more mild to moderate glaucoma patients, and increasingly even for more severe cases. The first United States Food and Drug Administration (FDA)-approved MIGS device, the iStent trabecular micro-bypass (containing one stent), and the recently FDA-approved second-generation iStent inject (containing two stents) are both designed to create patent pathways through the trabecular meshwork, which is known to be a major site of resistance to aqueous fluid outflow and is a major contributor to elevated IOP in glaucoma [21]. By bypassing the trabecular meshwork (particularly the juxtacanalicular tissue) and allowing direct aqueous flow into Schlemm's canal, the facility of outflow can be increased and consequently IOP can decrease.

Both devices have been studied in a variety of surgical settings: for example, in both standalone and cataract combination surgeries, in eyes with mild to severe glaucoma severity, in randomized controlled trials as well as independent surgeon case series, in different types of glaucoma [including pseudoexfoliative glaucoma (PXG) and normal-tension glaucoma (NTG)] as well as ocular hypertension, and in single- or multi-stent use [22-45]. These studies have consistently demonstrated sizable reductions in IOP and medication burden, while also maintaining a high safety profile and limited postoperative care. Importantly, the IOP and medication reductions appear durable over the long term, which is especially crucial in light of lengthening life spans, the chronic nature of the disease, and the need for a favorable cost-benefit ratio. Stent implantation also appears to delay or eliminate the need for more invasive surgery, thereby having the potential to further reduce healthcare burden and costs over the long term. Not surprisingly, given this treatment paradigm, the iStent and iStent inject devices have garnered several robust health economics analyses [8-14] which have demonstrated favorable cost-effectiveness across multiple countries and healthcare models.

In addition to the substantive evidence base supporting the use of either iStent or iStent inject, a comparative study published by Guedes et al. analyzed both devices and showed significantly greater IOP reduction and fewer adverse events with iStent inject versus iStent [45]. Additionally, while both devices resulted in significant medication reductions from baseline, iStent inject produced a greater reduction than iStent. Similarly, the present manuscript includes consecutive cases of both iStent or 
iStent inject implantation combined with cataract surgery. The cohort's inclusion of both implants within the same surgical setting and in the hands of the same surgeon allows us to evaluate each device without the confounding effects of different surgeons or different sites. To our knowledge, this is one of the first and largest datasets on the two devices in a single report, and it tracks outcomes through 1 year postoperatively.

\section{METHODS}

\section{Study Design and Participants}

This longitudinal retrospective study included all consecutive eyes implanted with either iStent or iStent inject with cataract surgery by a single glaucoma surgeon (DM) at a private practice in Australia. iStent surgeries took place from February 2014 to December 2015, and iStent inject surgeries occurred from February to December 2016. The choice of implant was based on availability in the Australian market.

Eligible patients had a diagnosis of glaucoma or ocular hypertension, cataract requiring surgery, follow-up data through 12 months postoperatively, and the need for IOP and/or medication reduction. Glaucoma diagnoses could include primary open-angle glaucoma (POAG), NTG, PXG, combined-mechanism glaucoma (CMG), and narrow-angle glaucoma (NAG; with open-angle configuration in area of stent implantation). Preoperative glaucoma severity was defined by visual field (VF) mean deviation using the Hodapp-Parrish-Anderson staging as mild ( 0 to $-6 \mathrm{~dB}$ ), moderate ( -6 to $-12 \mathrm{~dB}$ ), and severe (worse than $-12 \mathrm{~dB}$ ) [46].

The degree of effectiveness through 12 months was characterized by the mean IOP and glaucoma medication burden. Proportional analyses were completed for eyes achieving IOP $\leq 18 \mathrm{mmHg}, \quad$ IOP $\leq 15 \mathrm{mmHg}, \quad$ or $\quad$ IOP reduction $\geq 20 \%$ from preoperative; and eyes on 0 medications or $\geq 2$ medications. Safety outcomes included adverse events, secondary surgeries, VF, and best-corrected visual acuity (BCVA). IOP was measured by Goldmann applanation, and BCVA was measured using a standard Snellen chart. Proper positioning of the iStent and iStent inject was verified by gonioscopy examination, which was performed during surgery and at all clinical examinations throughout follow-up.

The chi-square test and Student's $t$ test were used for the analysis of categorical and numerical variables, respectively. A $p$ value of 0.05 was set as the threshold for statistical significance. All procedures were in accordance with the medical advisory committees of both Hunter Valley Private Hospital and Hunter Eye Hospital, and with the 1964 Declaration of Helsinki and its later amendments or comparable ethical standards. Given that this was a retrospective outcomes analysis, including only patients from the surgeon's real-world clinical population who already had received treatment, formal clinical trial registration was not required.

\section{Device and Surgical Technique}

The iStent and iStent inject trabecular microbypass devices are designed to create one or two patent bypasses, respectively, through the trabecular meshwork into Schlemm's canal. These bypasses facilitate aqueous outflow and thereby decrease IOP. The iStent trabecular micro-bypass consists of a single-use inserter preloaded with one biocompatible, non-ferromagnetic titanium stent having a length of $1.0 \mathrm{~mm}$, height of $0.33 \mathrm{~mm}$, and a "snorkel" bore diameter of $120 \mu \mathrm{m}$ (Fig. 1). The inserter is advanced $\mathrm{ab}$ internally through a small temporal clear corneal incision, thereby preserving ocular tissue in case other glaucoma surgery is needed in the future. The iStent inject trabecular microbypass consists of a single-use injector preloaded with two titanium stents, each having $230 \mu \mathrm{m}$ diameter, $360 \mu \mathrm{m}$ height, $80 \mu \mathrm{m}$ central lumen diameter, and four $50-\mu \mathrm{m}$ side outlets to allow for multidirectional outflow (Fig. 1). The stents are composed of biocompatible, non-ferromagnetic titanium, and each one is designed to carry the total amount of aqueous fluid produced by the human body (average $2.5 \mu \mathrm{l} / \mathrm{min}$ ). With these characteristics, two iStent inject stents have been shown to provide arcs of aqueous flow spanning up to 6 clock hours in 


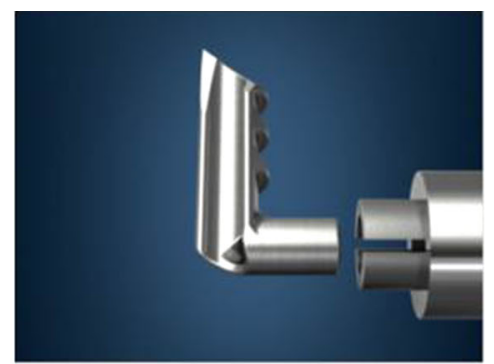

iStent $^{8}$

(1 stent per device)

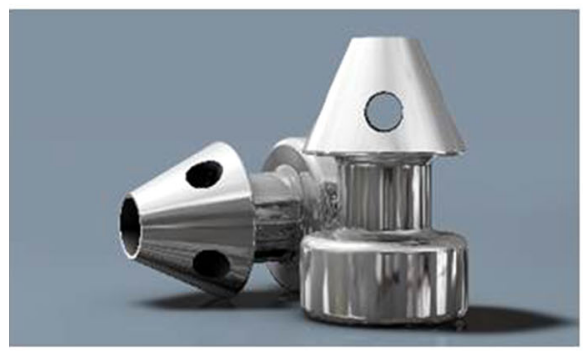

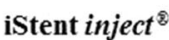

(2 stents per device, each with 4 lateral outlet lumens for multidirectional outflow)

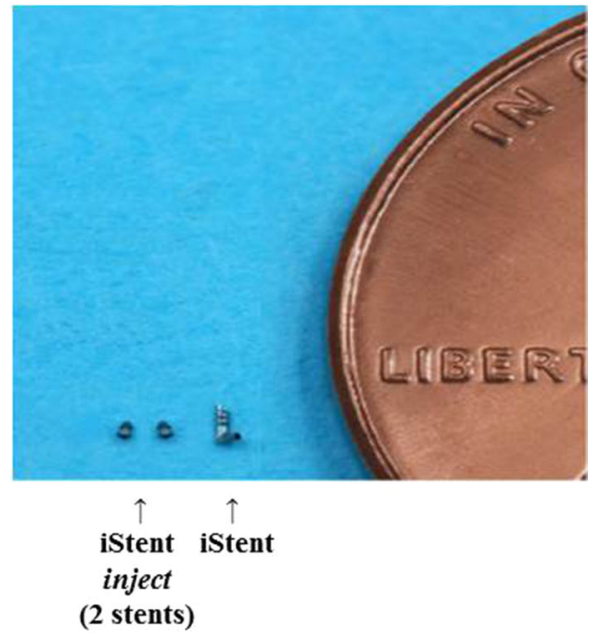

(2 stents)

Fig. 1 iStent ${ }^{\circledR}$ and iStent inject ${ }^{\circledR}$ trabecular micro-bypass stents, with relative dimensions (image courtesy of Glaukos Corp.)

the canal. To implant the stents, the surgeon advances the injector through the existing phacoemulsification incision (i.e. no additional incision needed), then positions both stents through the trabecular meshwork into the Schlemm's canal in a single ocular entry. After surgery, patients in this study were treated with topical antibiotic for 1 week and topical steroid tapered over 4 weeks.

\section{RESULTS}

\section{IOP and Medications}

A total of 137 consecutive eyes with mild to moderate glaucoma and cataract and 12 months of follow-up were included. The iStent group $(n=67)$ included predominantly eyes with POAG and PXG, and the iStent inject group $(n=70)$ included mostly eyes with POAG, ocular hypertension, and NAG (with open-angle configuration in area of stent implantation). Over $73 \%$ of eyes in both groups had early glaucoma, and approximately $22 \%$ of eyes had undergone prior glaucoma surgical or laser procedures. Complete preoperative parameters and demographics are shown in Table 1.

At 12 months postoperatively, mean IOP decreased from $18.4 \pm 4.2 \mathrm{mmHg}$ to $14.2 \pm$ $2.5 \mathrm{mmHg}$ in iStent eyes $(p<0.0001)$, and from $20.4 \pm 5.6 \mathrm{mmHg}$ to $14.4 \pm 2.1 \mathrm{mmHg}$ in iStent inject eyes $(p<0.0001)$. The IOP reduction was significantly greater for iStent inject eyes than iStent eyes $(6.0 \mathrm{mmHg}$ versus $4.2 \mathrm{mmHg}$ reduction, $p=0.034$ ) (Fig. 2). At 1 year after surgery, the iStent and iStent inject groups both had high proportions of patients achieving the IOP effectiveness endpoints, although there was a consistent trend toward greater proportions with iStent inject than iStent: IOP $\leq 18 \mathrm{mmHg}$ in $95.7 \%$ of iStent inject eyes and $92.5 \%$ of iStent eyes; IOP $\leq 15 \mathrm{mmHg}$ in $74.3 \%$ of iStent inject 
Table 1 Demographic and baseline ocular characteristics, iStent and iStent inject groups

\begin{tabular}{|c|c|c|}
\hline Characteristics & $\begin{array}{l}\text { iStent, } n=67 \\
\text { eyes of } 46 \\
\text { patients }\end{array}$ & $\begin{array}{l}\text { iStent inject, } \\
n=70 \text { eyes of } \\
42 \text { patients }\end{array}$ \\
\hline \multicolumn{3}{|l|}{ Age (years) } \\
\hline Mean \pm SD & $71.7 \pm 6.2$ & $70.3 \pm 7.2$ \\
\hline \multicolumn{3}{|l|}{ Gender } \\
\hline Male/female & $\begin{array}{l}30 \% / 70 \%(14 / \\
32)\end{array}$ & $\begin{array}{l}48 \% / 52 \%(20 / \\
22)\end{array}$ \\
\hline $\begin{array}{l}\text { Eyes with prior } \\
\text { glaucoma surgery or } \\
\text { laser procedures }\end{array}$ & $22.4 \%(15 / 67)$ & $21.4 \%(15 / 70)$ \\
\hline \multicolumn{3}{|c|}{ Visual field mean deviation $(\mathrm{dB})$} \\
\hline Mean $\pm S D$ & $-4.89 \pm 5.50$ & $-4.35 \pm 7.40$ \\
\hline \multicolumn{3}{|l|}{ Glaucoma type } \\
\hline POAG & $83.6 \%(56 / 67)$ & $64.3 \%(45 / 70)$ \\
\hline PXG & $11.9 \%(8 / 67)$ & $2.9 \%(2 / 70)$ \\
\hline $\mathrm{CMG}$ & $3.0 \%(2 / 67)$ & $0.0 \%(0 / 70)$ \\
\hline NTG & $1.5 \%(1 / 67)$ & $1.4 \%(1 / 70)$ \\
\hline OHT & $0.0 \%(0 / 67)$ & $20.0 \%(14 / 70)$ \\
\hline NAG & $0.0 \%(0 / 67)$ & $11.4 \%(8 / 70)$ \\
\hline \multicolumn{3}{|l|}{ Glaucoma stage $^{a}$} \\
\hline $\begin{array}{l}\text { Early glaucoma or } \\
\text { OHT }\end{array}$ & $73.5 \%$ & $80.3 \%$ \\
\hline Moderate & $14.3 \%$ & $9.1 \%$ \\
\hline Advanced & $12.2 \%$ & $10.6 \%$ \\
\hline $\begin{array}{l}\text { Baseline BCVA 20/30 } \\
\text { or better }\end{array}$ & $71.6 \%$ & $72.9 \%$ \\
\hline
\end{tabular}

$S D$ standard deviation, $O H T$ ocular hypertension

${ }^{a}$ Preoperative glaucoma severity was determined by VF mean deviation using the Hodapp-Parrish-Anderson staging as mild $(0 \geq \mathrm{VF} \quad \mathrm{MD} \geq-6 \mathrm{~dB})$, moderate $(-6>$ VF MD $\geq-12 \mathrm{~dB})$, and severe $(>-12 \mathrm{~dB})$

eyes and $71.6 \%$ of iStent eyes, and IOP decreased by $\geq 20 \%$ from preoperative in $68.6 \%$ of iStent inject eyes and $62.7 \%$ of iStent eyes (Fig. 3).

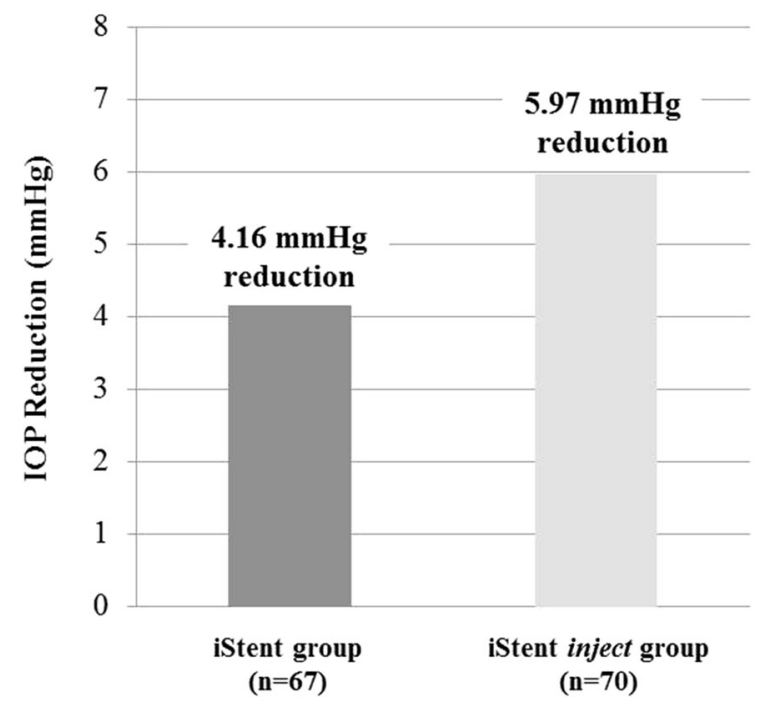

Fig. 2 Mean IOP reduction at 12 months postoperatively versus baseline*. iStent $(n=67)$ and iStent inject $(n=70)$. *IOP reduction was significantly greater for iStent inject eyes than iStent eyes $(5.97 \mathrm{mmHg}$ vs. $4.16 \mathrm{mmHg}$ reduction, $p=0.034)$. IOP $=$ intraocular pressure

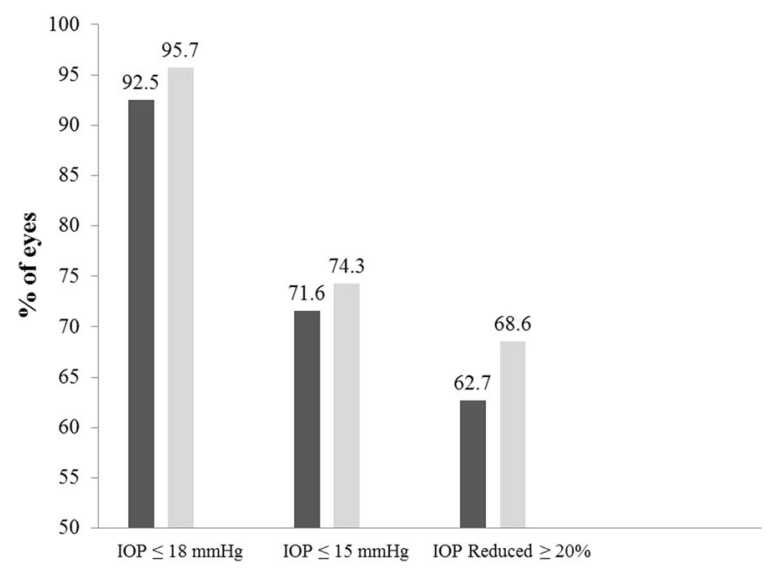

Fig. 3 Eyes achieving IOP $\leq 18 \mathrm{mmHg}$, IOP $\leq 15 \mathrm{mmHg}$, and IOP reduction $\geq 20 \%$ vs. baseline. iStent $(n=67)$ and iStent inject $(n=70)$. IOP intraocular pressure

Regarding medication usage, mean medication burden decreased from $1.8 \pm 0.7$ medications preoperatively to $0.3 \pm 0.5$ medications at 12 months in iStent eyes $(84.0 \%$ reduction, $p<0.0001$ ), and from $1.3 \pm 0.9$ medications preoperatively to $0.1 \pm 0.3$ medications at 12 months in iStent inject eyes $(94.7 \%$ 


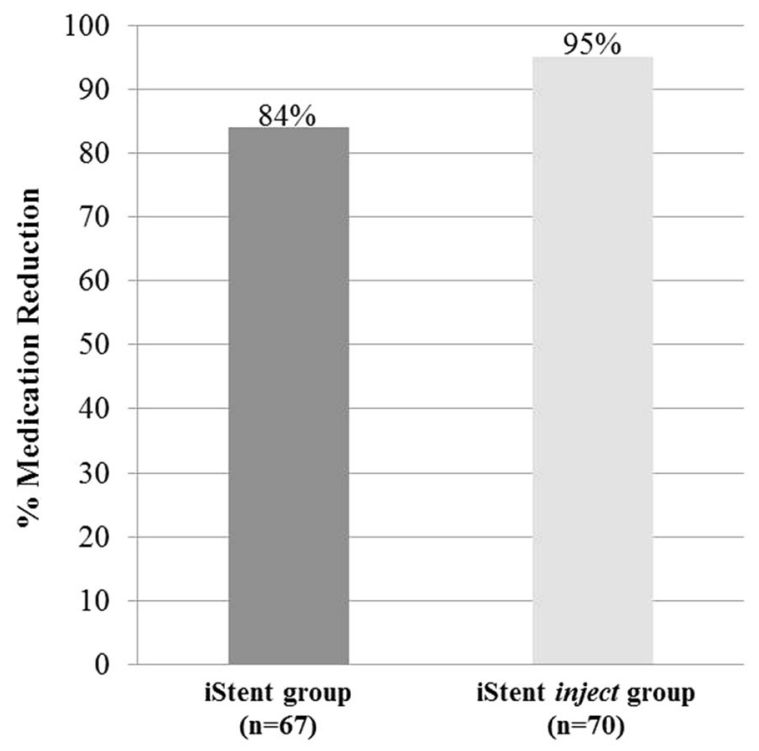

Fig. 4 Medication reduction at 12 months postoperatively vs. baseline*. iStent $(n=67)$ and iStent inject $(n=70)$. ${ }^{*}$ Medication reduction was significant in each group ( $p<0.0001$ for both). Med medication

reduction, $p<0.0001$ ) (Fig. 4). All patients in both groups were able to maintain or decrease their number of medications versus preoperative. The percentage of eyes on 2 or more medications decreased significantly in both groups: from $68.7 \%$ preoperatively to $4.5 \%$ at 1 year in the iStent group $(p<0.0001)$, and from $41.4 \%$ preoperatively to $0 \%$ at 1 year in the iStent inject group $(p<0.0001)$ (Fig. 5). A significantly higher proportion of iStent inject eyes than iStent eyes were medication-free at 12 months $(92.9 \%$ versus $76.1 \%$ in the two groups, respectively; $p=0.0068$ ) (Fig. 5).

\section{Safety}

No intraoperative complications occurred in either group. All iStent eyes had successful implantation of one stent. All but two iStent inject eyes had successful implantation of two stents; the remaining two eyes had only one stent implanted, but did not experience any associated sequelae or complications; final IOP was $14 \mathrm{mmHg}$ and $19 \mathrm{mmHg}$ on one medication in each of these two eyes. At 3 months postoperatively, one iStent eye had a corneal erosion (unrelated to stenting) which was treated successfully with a bandage contact lens and eye drops and resulted in no subsequent sequelae or recurrence. No adverse events occurred in iStent inject eyes. There were no reports of persistent intraocular inflammation or peripheral anterior synechiae (PAS) in either group. No eye in either group underwent a secondary glaucoma surgery during the followup period.

Gonioscopy was performed intraoperatively and at all postoperative examinations; no significant problems in stent position were identified in either group at any point during the study. Through 12 months postoperatively, visual acuity remained stable or improved in both groups, consistent with expectations for phacoemulsification cataract surgery. Visual acuity was $20 / 30$ or better in $71.6 \%$ of iStent eyes preoperatively, rising to $98.5 \%$ of iStent eyes at 12 months postoperatively; in iStent inject eyes, the percentage with $20 / 30$ or better vision rose from $72.9 \%$ preoperatively to $95.7 \%$ at 12 months. Visual fields remained stable in both groups: mean deviation of $-4.89 \mathrm{~dB}$ preoperatively versus $-4.75 \mathrm{~dB}$ at 1 year in iStent eyes ( $p=0.8816$, not significant); and $-4.35 \mathrm{~dB}$ preoperatively versus $-4.42 \mathrm{~dB}$ at 1 year in iStent inject eyes ( $p=0.9536$, not significant).

\section{DISCUSSION}

This retrospective consecutive case series examined iStent or iStent inject trabecular micro-bypass device implantation in conjunction with cataract surgery in a real-world patient population with mild to moderate glaucoma. This represents one of the first and largest datasets evaluating both devices in the hands of a single surgeon at a single surgical site. Both devices produced significant reductions in IOP and medication burden through 1 year postoperatively, with final IOP under $14.5 \mathrm{mmHg}$ and medication burden decreased by $84-95 \%$ in both groups. Although the study was not designed for prospective comparison, several trends did emerge that began to distinguish the two groups. IOP reduction was significantly 


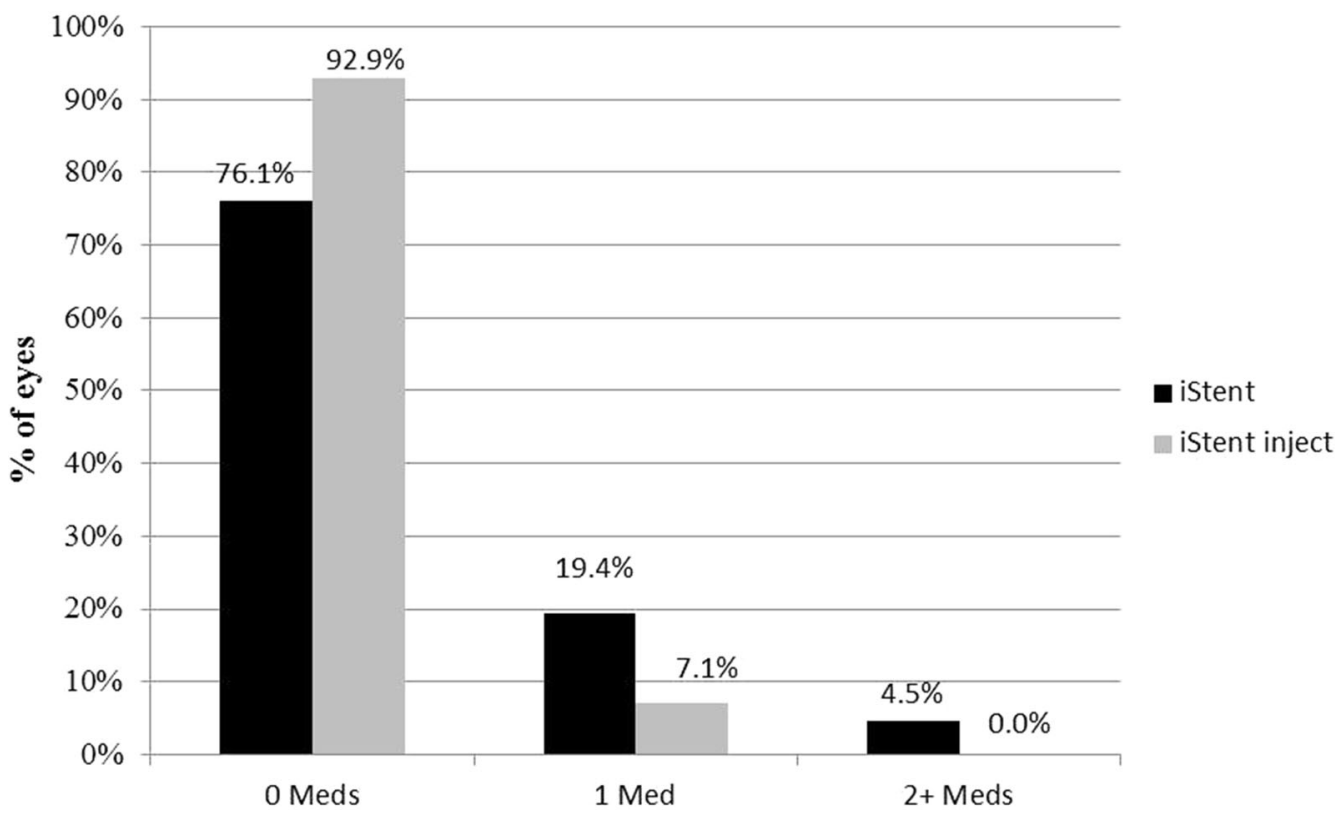

Fig. 5 Proportional analysis of medication burden at 12 months postoperatively*. iStent $(n=67)$ and iStent inject $(n=70) .{ }^{*} \mathrm{~A}$ significantly higher proportion of iStent inject

greater in iStent inject eyes than iStent eyes at 1 year $(6.0 \mathrm{mmHg}$ versus $4.2 \mathrm{mmHg}$ reduction, respectively), and there was a consistent trend toward greater proportions of iStent inject eyes achieving the effectiveness endpoints of IOP $\leq 18 \mathrm{mmHg}, \quad$ IOP $\leq 15 \mathrm{mmHg}$, and IOP reduction $\geq 20 \%$ versus baseline. Furthermore, a significantly greater proportion of iStent inject eyes than iStent eyes were medication-free at 12 months $(92.9 \%$ versus $76.1 \%$ medicationfree, respectively), and the percent reduction in medications from baseline was greater in iStent inject eyes than iStent eyes $(94.7 \%$ versus $84.0 \%$ reduction, respectively).

In terms of absolute medication reduction, both groups were able to eliminate 1-1.5 glaucoma medications from their existing regimens. This reduction has substantial benefits for the patient and the healthcare system, given the well-known downsides of medications such as ocular surface damage, local and systemic side effects, financial and social costs, and poor treatment adherence [2-16, 47-51]. Adherence is widely known to be critical in achieving consistent IOP lowering in order to minimize eyes were medication-free at 12 months than iStent eyes (92.9\% versus $76.1 \%$ in the two groups, respectively; $p=0.0068)$. Med medication

the risk of optic nerve damage and glaucoma progression [47]. Lower medication burden may substantially improve patient adherence, particularly in patients with multiple medications, given that adherence is known to dramatically decline with multiple versus single eye drops [47]. Our study's findings are particularly meaningful in light of these latter observations about adherence: specifically, that $0 \%$ of iStent inject eyes were on multiple medications at 12 months, and $92.9 \%$ were medication-free. Furthermore, this significant medication reduction was accompanied by an IOP reduction of almost $6 \mathrm{mmHg}$ versus baseline, which was significantly greater than the $4.2-\mathrm{mmHg}$ IOP reduction resulting from iStent, thereby corroborating the comparative benefit of iStent inject in reducing both IOP and medication in glaucoma patients.

Some of these trends in effectiveness outcomes between the iStent and iStent inject groups may point to underlying differences between the two devices. Three key characteristics are thought to favorably distinguish the iStent inject device over the iStent, as cited by 
Guedes et al. in a recent real-world case series [45]. The iStent inject device contains two preloaded stents (instead of the iStent's one stent) in a single injector, thereby bypassing two separate regions of the trabecular meshwork and enabling up to 6 clock hours of aqueous egress. Each of these stents is capable of carrying 2.5 $\mu \mathrm{L} / \mathrm{min}$ of aqueous humor, the maximum amount typically produced by the human body. Second, each stent has four outlet lumens (rather than one), in order to provide multidirectional flow and maximize the number of accessible collector channels. Third, the iStent inject stents are smaller and designed for more direct implantation into Schlemm's canal, allowing for greater procedural efficiency which in turn may reduce complications, increase the proportion of first-attempt successful implantations, and hasten implementation into a doctor's surgical practice.

The two-stent, multidirectional design of the iStent inject is backed up by robust research in aqueous angiography and computational fluid dynamics (CFD), the most powerful tool available to assess size and flow. In a study by Huang et al., significant aqueous angiographic outflow improvement resulted after iStent inject implantation with cataract surgery [52]. The findings showed reactivation of formerly dormant outflow areas and possible access to up to 6 clock hours of collector channels for aqueous humor outflow. Despite the micro-size of either iStent or iStent inject, analytical approximations supported by CFD modeling indicated that each stent presented a negligible flow resistance that resulted in significant IOP reduction. State-of-the-art CFD models estimated that a single iStent or iStent inject was wholly sufficient to conduct the entire $2.5 \mathrm{uL} /$ min of aqueous humor production [53].

The use of two versus one stent is backed by preclinical and clinical studies evaluating changes in IOP and outflow facility after implantation of single or multiple trabecular micro-bypass stents. These studies demonstrated that efficacy is enhanced when combining two devices compared to a single device. Using an anterior segment perfusion model, Bahler et al. showed that IOP decreased from 21.4 to $12.4 \mathrm{mmHg}$ after implantation of one
iStent $(p<0.001)$; eyes receiving more than one stent had final IOP of $11.9 \mathrm{mmHg}$ [54]. In a second study, a single iStent inject stent increased outflow facility from 0.16 to $0.38 \mu \mathrm{L} /$ $\mathrm{min} / \mathrm{mmHg}(p<0.03, n=7)$, and IOP concurrently decreased from 16.7 to $8.6 \mathrm{mmHg}$, while addition of a second iStent inject stent further increased outflow facility to $0.78 \mu \mathrm{L} / \mathrm{min} /$ $\mathrm{mmHg}(n=2)$ [55]. Similarly, Hunter et al., using whole-eye perfusion models, showed $6.0 \mathrm{mmHg}$ of IOP reduction versus baseline with a single iStent, and an additional $2.9 \mathrm{mmHg}$ of IOP reduction with a second iStent, for a total IOP reduction of $8.9 \mathrm{mmHg}$ from baseline [53].

On the clinical side, a prospective randomized trial by Katz et al. compared long-term outcomes following implantation of one, two, and three iStents in a standalone procedure, and Belovay et al. and El Wardani et al. evaluated two or three iStents combined with cataract surgery $[33,56,57]$. All three clinical studies demonstrated that the majority of IOP reduction occurs after the first stent and that additional stents produce further IOP reduction, likely due to access to more regions of the distal outflow network and increased probability of stenting areas of Schlemm's canal that are still patent and functional. The outcomes suggest that iStent and iStent inject treatment is titratable, allowing lower IOP to be achieved with additional stent implantation.

The safety profile was highly favorable in both groups, including no stent-related adverse events and no secondary glaucoma surgeries. The device is made of biocompatible material and notably does not contain nickel, which is widely known to be pro-inflammatory and has warranted restrictive regulations in the European Union [58-66]. Nickel allergy is present in an estimated $8-19 \%$ of the general population [59], most of whom are not aware of the allergy, which is a real concern for a permanent implanted device. For example, joint replacements containing nickel have been associated with poor wound healing, recurrent joint effusion, pain, and implant loosening [63], while neurosurgical nickel-containing aneurysm clips have been implicated in life-threatening strokes and seizure $[67,68]$. Not surprisingly given the 
biocompatible, nickel-free makeup of the iStent and iStent inject devices, there were no cases of intraocular inflammation or PAS in either group throughout the course of follow-up; this is in contrast to the reported PAS rates of up to $30 \%$ observed in trials of other MIGS devices with similar mechanism of action [69-72]. This corroborates the existing literature showing high safety profiles for these stents [22-45]. Visual acuity was stable or improved, indicating that stent implantation did not detract from the visual improvements expected after cataract surgery. Visual fields also remained stable during the study, and are continuing to be monitored to evaluate for longer-term stability. There were no cases of any of the complications typically seen with traditional filtering surgeries such as choroidal detachment, bleb-related complications, endophthalmitis, or hypotony [18-20]. There also were no cases of choroidal detachment or folds, device dislocation, hypotony, significant hyphema, or corneal endothelial cell compromise such as that cited with some other MIGS devices [73-76].

Certain limitations should be discussed in the context of this retrospective, non-randomized case series. The study included all-comers, rather than employing strict eligibility criteria customary in formal clinical trials. Since cases were drawn consecutively from the surgeon's real-life clinical population, the groups were not prospectively matched for ocular parameters, and their outcomes were not intended for formal direct comparison-although notable preliminary trends indeed could be observed. A strength of the study is the absence of intersurgeon variability given that this was singlesurgeon data; however, this also could limit generalizability to broader populations. The report sought to augment the information available to the ophthalmic community about the two technologies; future research could expand upon this knowledge via prospective comparative clinical trials, trials with larger sample sizes, multicenter trials, or analyses of different glaucoma subtypes. No medication washouts were completed, and there was no control group of cataract surgery only, as these would not be appropriate in this real-world setting. However, IOP and medications are numeric values, and all examinations and medication decisions were completed by the same surgeon throughout, so patients' preoperative measurements could reasonably act as their own controls. As iStent inject is a relatively new technology in many regions of the world, this report providing 1-year data is valuable given the need for comparative iStent versus iStent inject studies; patient follow-up is ongoing so that longer-term outcomes can be assessed.

\section{CONCLUSIONS}

In conclusion, this retrospective analysis provides data on both iStent and iStent inject in eyes with glaucoma. Outcomes through 12 months showed clinically and statistically significant reductions in IOP and medication, while maintaining excellent safety. Although this retrospective study was not designed for direct comparison of the two devices, some consistent directional trends did become apparent-trends which suggested significantly greater efficacy with the iStent inject versus the iStent. These findings were achieved in a realworld setting and included consecutive patients, thereby promoting generalizability to other ophthalmic practices.

\section{ACKNOWLEDGEMENTS}

We thank the participants of the study.

Funding. No financial sponsorship was received for the work in this study; the patients were seen as part of Dr. Manning's normal clinical practice. The Rapid Service Fees were funded by Glaukos Corporation. Dr. Manning had full access to all of the data in this study and takes complete responsibility for the integrity of the data and accuracy of the data analysis.

Medical Writing and/or Editorial Assistance. Editorial assistance was provided and funded by Glaukos Corporation. 
Authorship. All named authors meet the International Committee of Medical Journal Editors (ICMJE) criteria for authorship for this article, take responsibility for the integrity of the work as a whole, and have given their approval for this version to be published.

Disclosures. Dr. David Manning is a speaker and member of the advisory board for Glaukos and is a member of the advisory board for Alcon and Allergan.

Compliance with Ethics Guidelines. All procedures performed were in accordance with the ethical standards of the Ethics Committee of the Hunter New England Local Health District and with the 1964 Declaration of Helsinki and its later amendments or comparable ethical standards.

Data Availability. The datasets generated and/or analyzed during the current study are available from the corresponding author on reasonable request.

Open Access. This article is distributed under the terms of the Creative Commons Attribution-NonCommercial 4.0 International License (http://creativecommons.org/licenses/ by-nc/4.0/), which permits any noncommercial use, distribution, and reproduction in any medium, provided you give appropriate credit to the original author(s) and the source, provide a link to the Creative Commons license, and indicate if changes were made.

\section{REFERENCES}

1. Quigley HA, Broman AT. The number of people with glaucoma worldwide in 2010 and 2020. Br J Ophthalmol. 2006;90(3):262-7.

2. Stewart WC, Konstas AG, Nelson LA, Kruft B. Metaanalysis of 24-hour intraocular pressure studies evaluating the efficacy of glaucoma medicines. Ophthalmology. 2008;115:1117-22.

3. Bhosle MJ, Reardon G, Camacho FT, et al. Medication adherence and health care costs with the introduction of latanoprost therapy for glaucoma in a Medicare managed care population. Am J Geriatr Pharmacother. 2007;5:100-11.

4. Boland MV, Ervin AM, Friedman DS, et al. Comparative effectiveness of treatments for open-angle glaucoma: a systematic review for the U.S. Preventive Services Task Force. Ann Intern Med. 2013;158:271-9.

5. Baudouin C, Labbé A, Liang H, Pauly A, BrignoleBaudouin F. Preservatives in eyedrops: the good, the bad and the ugly. Prog Retin Eye Res. 2010;29(4):312-34.

6. Zhang X, Vadoothker S, Munir WM, Saeedi O. Ocular surface disease and glaucoma medications: a clinical approach. Eye Contact Lens. 2019; 45(1):11-8.

7. Inoue K. Managing adverse effects of glaucoma medications. Clin Ophthalmol. 2014;8:903-13.

8. Tan SZ, Au L. Manchester iStent study: 3-year results and cost analysis. Eye. 2016;30:1-6.

9. Berdahl JP, Khatana AK, Katz LJ, et al. Cost-comparison of two trabecular micro-bypass stents versus selective laser trabeculoplasty or medications only for intraocular pressure control for patients with open-angle glaucoma. J Med Econ. 2017;20(7):760-6.

10. Wang SY, Singh K, Stein JD, Chang RT. Ocular antihypertensive medication use after iStent implantation concurrent with cataract surgery vs cataract surgery alone in a large US health care claims database. JAMA Ophthalmol. 2018. https:// doi.org/10.1001/jamaophthalmol.2018.4461.

11. Ordóñez JE, Ordóñez A, Osorio UM. Cost-effectiveness analysis of iStent trabecular micro-bypass stent for patients with open-angle glaucoma in Colombia. Curr Med Res Opin. 2018;10:1-12.

12. Ngan K, Fraser E, Buller S, Buller A. A cost minimisation analysis comparing iStent accompanying cataract surgery and selective laser trabeculoplasty versus topical glaucoma medications in a public healthcare setting in New Zealand. Graefes Arch Clin Exp Ophthalmol. 2018;256(11):2181-9.

13. Patel V, Ahmed I, Podbielski D, Falvey H, Murray J, Goeree R. Cost-effectiveness analysis of standalone trabecular micro-bypass stents in patients with mild-to-moderate open-angle glaucoma in Canada. J Med Econ. 2019;22(4):390-401.

14. Iordanous Y, Kent JS, Hutnik CM, Malvankar-Mehta MS. Projected cost comparison of Trabectome, iStent, and endoscopic cyclophotocoagulation versus glaucoma medication in the Ontario Health Insurance Plan. J Glaucoma. 2014;23(2):e112-8. 
15. Olthoff CM, Schouten JS, van de Borne BW, Webers CA. Noncompliance with ocular hypotensive treatment in patients with glaucoma or ocular hypertension an evidence-based review. Ophthalmology. 2005;112:953-61.

16. Schwartz GF, Quigley HA. Adherence and persistence with glaucoma therapy. Surv Ophthalmol. 2008;53(Suppl 1):S57-68.

17. Glaucoma Laser Trial Research Group. The glaucoma laser trial (GLT) and glaucoma laser trial follow-up study: 7. Results. Am J Ophthalmol. 1995;120:718-31.

18. Jampel HD, Musch DC, Gillespie BW, Lichter PR, Wright MM, Guire KE, Collaborative Initial Glaucoma Treatment Study Group. Collaborative Initial Glaucoma Treatment Study Group. Perioperative complications of trabeculectomy in the collaborative initial glaucoma treatment study (CIGTS). Am J Ophthalmol. 2005;140(1):16-22.

19. Rulli E, Biagioli E, Riva I, et al. Efficacy and safety of trabeculectomy vs nonpenetrating surgical procedures: a systematic review and meta-analysis. JAMA Ophthalmol. 2013;131(12):1573-82.

20. Gedde SJ, Herndon LW, Brandt JD, Budenz DL, Feuer WJ, Schiffman JC, Tube Versus Trabeculectomy Study Group. Postoperative complications in the tube versus trabeculectomy (TVT) study during five years of follow-up. Am J Ophthalmol. 2012;153:804-14.

21. Grant WM. Experimental aqueous perfusion in enucleated human eyes. Arch Ophthalmol. 1963; 69:783-801.

22. Samuelson TW, Katz LJ, Wells JM, et al. Randomized evaluation of the trabecular micro-bypass stent with phacoemulsification in patients with glaucoma and cataract. Ophthalmol. 2011;118:459-67.

23. Craven ER, Katz LJ, Wells JM, Giamporcaro JE, iStent Study Group. Cataract surgery with trabecular micro-bypass stent implantation in patients with mild-to-moderate open-angle glaucoma and cataract: two-year follow-up. J Cataract Refract Surg. 2012;38(8):1339-45

24. Arriola-Villalobos P, Martinez-de-la-Casa JM, DiazValle D, et al. Combined iStent trabecular microbypass stent implantation and phacoemulsification for coexisting open-angle glaucoma and cataract: a long-term study. Br J Ophthalmol. 2012;96:645-9.

25. Fea AM, Consolandi G, Zola M, et al. Micro-bypass implantation for primary open-angle glaucoma combined with phacoemulsification: 4-year followup. J Ophthalmology. 2015;26(2015):795357.
26. Gallardo MJ, Supnet RA, Giamporcaro JE, Hornbeak DM. Outcomes of combined trabecular micro-bypass and phacoemulsification in a predominantly Hispanic patient population. Clin Ophthalmol. 2016;10:1931-7.

27. Neuhann TH. Trabecular micro-bypass stent implantation during small incision cataract surgery for open angle glaucoma or ocular hypertension: long term results. J Cataract Refract Surg. $2015 ; 41: 2664-71$.

28. Ferguson T, Swan R, Ibach M, Schweitzer J, Sudhagoni R, Berdahl JP. Evaluation of a trabecular microbypass stent with cataract extraction in severe primary open-angle glaucoma. J Glaucoma. 2018;27(1):71-6.

29. Ferguson TJ, Swan R, Ibach M, Schweitzer J, Sudhagoni R, Berdahl JP. Trabecular microbypass stent implantation with cataract extraction in pseudoexfoliation glaucoma. J Cataract Refract Surg. 2017;43(5):622-6.

30. Ferguson TJ, Berdakl JP, Schweitzer JA, Sudhagoni RG. Clinical evaluation of a trabecular micro-bypass stent with phacoemulsification in patients with open-angle glaucoma and cataract. Clin Ophthamol. 2016;10:1767-73.

31. Ferguson T, Berdahl J, Schweitzer J, Sudhagoni R. Evaluation of a trabecular micro-bypass stent in pseudophakic patients with open-angle glaucoma. J Glaucoma. 2016;25:896-900.

32. Fechtner RD, Voskanyan L, Vold SD, et al. Five-year, prospective, randomized, multi-surgeon trial of two trabecular bypass stents versus prostaglandin for newly-diagnosed open-angle glaucoma. Ophthalmol Glaucoma. 2019;2(3):156-66.

33. Katz LJ, Erb C, Carceller Guillamet A, et al. Longterm titrated IOP control with one, two or three trabecular micro-bypass stents in open-angle glaucoma subjects on topical hypotensive medication: 42-month outcomes. Clin Ophthalmol. 2018; $12: 255-62$.

34. Donnenfeld ED, Solomon KD, Voskanyan L, et al. A prospective 3-year follow-up trial of implantation of two trabecular microbypass stents in open-angle glaucoma. Clin Ophthalmol. 2015;9:2057-65.

35. Chang DF, Donnenfeld ED, Katz LJ, et al. Efficacy of two trabecular micro-bypass stents combined with topical travoprost in open-angle glaucoma not controlled on two preoperative medications: 3-year follow-up. Clin Ophthalmol. 2017;11:523-8.

36. Samuelson TW, Sarkisian SR Jr, Lubeck DM, for the iStent inject Study Group, et al. Prospective, randomized, controlled pivotal trial of iStent inject 
trabecular micro-bypass in primary open-angle glaucoma and cataract: two-year results. Ophthalmology. 2019;126(6):811-21.

37. Fea AM, Belda JI, Rękas M, et al. Prospective unmasked randomized evaluation of the iStent inject $^{\circledR}$ versus two ocular hypotensive agents in patients with primary open- angle glaucoma. Clin Ophthalmol. 2014;8:875-82.

38. Voskanyan L, García-Feijoo J, Belda JI, Fea A, Jünemann A, Baudouin C. Synergy Study Group. Prospective, unmasked evaluation of the iStent ${ }^{\circledR}$ inject system for open-angle glaucoma: Synergy Trial. Adv Ther. 2014;31(2):189-201.

39. Arriola-Villalobos P, Martinez-de-la-Casa JM, DiazValle D, Morales-Fernandez L, Fernandez-Perez C, Garcia-Feijoo J. Glaukos iStent inject ${ }^{\circledR}$ trabecular micro-bypass implantation associated with cataract surgery in patients with coexisting cataract and open-angle glaucoma or ocular hypertension: a long-term study. J Ophthalmol. 2016;2016: 1056573.

40. Klamann MK, Gonnermann J, Pahlitzsch M, et al. iStent inject in phakic open angle glaucoma. Graefes Arch Clin Exp Ophthalmol. 2015;253:941-7.

41. Berdahl J, Voskanyan L, Myers JS, et al. Implantation of two second-generation trabecular microbypass stents and topical travoprost in open-angle glaucoma not controlled on two preoperative medications: 18-month follow-up. Clin Exp Ophthalmol. 2017;45(8):797-802.

42. Lindstrom R, Lewis R, Hornbeak DM, et al. Outcomes following implantation of two second-generation trabecular micro-bypass stents in patients with open-angle glaucoma on one medication: 18-month follow-up. Adv Ther. 2016;33(11): 2082-90.

43. Hengerer FH, Auffarth GU, Riffel C, Conrad-Hengerer I. Prospective, non-randomized, 36-month study of second-generation trabecular micro-bypass stents with phacoemulsification in various types of glaucoma. Ophthalmol Ther. 2018;7(2):405-15.

44. Hengerer FH, Auffarth GU, Riffel C, Conrad-Hengerer I. Second-generation trabecular micro-bypass stents as standalone treatment for glaucoma: a 36-month prospective study. Adv Ther. 2019;36(7): 1606-17.

45. Guedes RAP, Gravina DM, Lake JC, Guedes VMP, Chaoubah A. Intermediate results of iStent or iStent inject implantation combined with cataract surgery in a real-world setting: a longitudinal retrospective study. Ophthalmol Ther. 2019;8(1):87-100.
46. Hodapp E, Parrish RK II, Anderson DR. Clinical decisions in glaucoma. St Louis: The CV Mosby Co; 1993.

47. Robin AL, Covert D. Does adjunctive glaucoma therapy affect adherence to the initial primary therapy? Ophthalmology. 2005;112:863-8.

48. Nordstrom BL, Friedman DS, Mozaffari E, Quigley HA, Walker AM. Persistence and adherence with topical glaucoma therapy. Am J Ophthalmol. 2005;140(4):598-606.

49. Newman-Casey PA, Robin AL, Blachley T, et al. The most common barriers to glaucoma medication adherence: a cross-sectional survey. Ophthalmology. 2015;122(7):1308-16.

50. Tsai JC. A comprehensive perspective on patient adherence to topical glaucoma therapy. Ophthalmology. 2009;116:S30-6.

51. Leung EW, Medeiros FA, Weinreb RN. Prevalence of ocular surface disease in glaucoma patients. J Glaucoma. 2008;17(5):350-5.

52. Huang AS, Penteado RC, Papoyan V, Voskanyan L, Weinreb RN. Aqueous angiographic outflow improvement after trabecular micro-bypass in glaucoma patients. Ophthalmol Glaucoma. 2019;2:11-21.

53. Hunter K, Fjield T, Heitzmann H, Shandas R, Kahook M. Characterization of micro-invasive trabecular bypass stents by ex vivo perfusion and computational flow modeling. Clin Ophthalmol. 2014;8:499-506.

54. Bahler CK, Smedley GT, Zhou J, Johnson DH. Trabecular bypass stents decrease intraocular pressure in cultured human anterior segments. Am J Ophthal. 2004;138(6):988-94.

55. Bahler C, Hann C, Fjield T, Haffner D, Heitzmann $\mathrm{H}$, Fautsch MP. Second-generation trabecular meshwork bypass stent (iStent inject) increases outflow facility in cultured human anterior segments. Am J Ophthal. 2012;153(6):1206-13.

56. Belovay GW, Naqi A, Chan BJ, Rateb M, Ahmed II. Using multiple trabecular micro-bypass stents in cataract patients to treat open-angle glaucoma. J Cataract Refract Surg. 2012;38(11):1911-7.

57. El Wardani M, Bergin C, Achache F, Sharkawi E. Evaluating the trabecular micro-bypass stent combined with phacoemulsification compared to phacoemulsification alone. Klin Monatsbl Augenheilkd. 2015;232:442-5.

58. Ahlström MG, Menné T, Thyssen JP, Johansen JD. The European nickel regulation and changes since 
introduction. Contact Dermatitis 2017:76: 382-384. Erratum in: Contact Dermatitis 2017: 77: 64-65.

59. Ahlström MG, Thyssen JP, Menné T, Johansen JD. Prevalence of nickel allergy in Europe following the EU Nickel Directive-a review. Contact Dermatitis. 2017;77(4):193-200.

60. Tsang ACO, Nicholson P, Pereira VM. Nickel-related adverse reactions in the treatment of cerebral aneurysms: a literature review. World Neurosurg. 2018;115:147-53.

61. Pacheco KA. Allergy to surgical implants. Clin Rev Allergy Immunol. 2019;56(1):72-85.

62. Saito M, Arakaki R, Yamada A, Tsunematsu T, Kudo Y, Ishimaru N. Molecular mechanisms of nickel allergy. Int J Mol Sci. 2016;17(2):E202.

63. Thomas P, Summer B. Implant allergy. Allergol Select. 2017;1(1):59-64.

64. Univers J, Long C, Tonks SA, Freeman MB. Systemic hypersensitivity reaction to endovascular stainless steel stent. J Vasc Surg. 2018;67(2):615-7.

65. Jacob SE, Goldenberg A, Pelletier JL, Fonacier LS, Usatine R, Silverberg N. Nickel allergy and our children's health: a review of indexed cases and a view of future prevention. Pediatr Dermatol. 2015;32(6):779-85.

66. Kręcisz B, Kieć-Świerczyńska M, ChomiczewskaSkóra D. Allergy to orthopedic metal implants-a prospective study. Int J Occup Med Environ Health. 2012;25(4):463-9.

67. Grande A, Grewal S, Tackla R, Ringer AJ. Lifethreatening allergic vasculitis after clipping an unruptured aneurysm: case report, weighing the risk of nickel allergy. Surg Neurol Int. 2014;5(Suppl 4):S161-4.

68. Schmidlin K, Verzwyvelt J, Bernstein D, Kim H. Probable delayed-type hypersensitivity to nickelcontaining cerebral aneurysm clip associated with neurologic deficits. J Allergy Clin Immunol Pract. 2015;3:609-11.

69. Pfeiffer N, Garcia-Feijoo J, Martinez-de-la-Casa JM, et al. A randomized trial of a Schlemm's canal microstent with phacoemulsification for reducing intraocular pressure in open-angle glaucoma. Ophthalmology. 2015;122:1283-93.

70. Gandolfi SA, Ungaro N, Ghirardini S, et al. Comparison of surgical outcomes between canaloplasty and Schlemm's canal scaffold at 24 months' follow up. J Ophthalmol. 2016;2016:3410469.

71. Samuelson TW, Chang DF, Marquis R, HORIZON Investigators, et al. A Schlemm canal microstent for intraocular pressure reduction in primary open-angle glaucoma and cataract: the HORIZON study. Ophthalmology. 2019;126(1):29-37.

72. Fea A. 36 month results from a multicenter randomised study of a Schlemm's canal microstent for reduction of IOP in primary open-angle glaucoma: the HYDRUS II trial. Presented at XXXVI Congress of the European Society of Cataract and Refractive Surgeons (ESCRS); September 22-26, 2018;Vienna, Austria.

73. Gillmann K, Bravetti GE, Mermoud A, Mansouri K. Anterior chamber XEN gel stent movements: the impact on corneal endothelial cell density. J Glaucoma. 2019;28(6):e93-5.

74. Buffault J, Baudouin C, Labbé A. $X N^{\circledR}$ gel stent for management of chronic open angle glaucoma: a review of the literature. J Fr Ophtalmol. 2019;42(2):e37-46.

75. Minckler DS, Baerveldt G, Alfaro MR, Francis BA. Clinical results with the trabectome for treatment of open-angle glaucoma. Ophthalmology. 2005;112(6):962-7.

76. Sieck EG, Epstein RS, Kennedy JB, et al. Outcomes of kahook dual blade goniotomy with and without phacoemulsification cataract extraction. Ophthalmol Glaucoma. 2018;1:75-81. 